\title{
The Psychometric Properties of the Persian Version of the Pregnancy Physical Activity Questionnaire
}

\author{
Azita Fathnezhad Kazemi ${ }^{1}$, Sepideh Hajian ${ }^{2 *}{ }^{(\mathbb{D}}$, Nasibeh Sharifi $^{3}$
}

\begin{abstract}
Objectives: Evidence indicates that regular physical activity has positive effects on the mother's health and outcomes of pregnancy. The measurement of physical activity during pregnancy is required for planning and developing health-related interventions. The aim of this study was to evaluate the psychometric properties of the Persian version of the pregnancy physical activity questionnaire. Materials and Methods: This cross-sectional methodological study was conducted with a sample of 200 pregnant women living in Tabriz. Women with low-risk singleton pregnancies in the age range of 20-40 years were included in the study. Sampling was carried out using a multistage sampling method in healthcare centers. Exploratory factor analysis (principal component analysis with varimax rotation), multivariate analysis of variance and Pearson correlation were used for data analysis. Reliability and internal consistency of the questionnaire were assessed using a test-retest method with a 2-week interval and Cronbach's alpha coefficient, respectively.

Results: A five-factor model was developed: 'vulnerable people', 'activities related to work', 'home', 'sports' and 'activities related to leisure time.' They accounted for $43.32 \%$ of the observed variance. The Cronbach's alpha coefficients of the entire instrument and domains were 0.879 and $0.83-0.93$, respectively. The Intra-class correlation coefficient (ICC) was equal to 0.857 (CI95\% $=0.75-0.93$ ) indicating proper stability of the questionnaire over time.

Conclusions: The Persian version of the pregnancy physical activity questionnaire is a valid and reliable tool for examining the risk of physical activities among Iranian pregnant women.

Keywords: Pregnancy, Physical activity, Psychometric properties.
\end{abstract}

\section{Introduction}

Physical activity is known as an element of health promoting behaviors, preventing and improving factor of health-related issues (1). Failure to engage in physical activity is a major risk factor for obesity and cardiovascular diseases (2). In spite of the emphasis on doing exercise in both genders $(3,4)$, women participate in physical activities less than men. Therefore, they are more susceptible to immobilization (5). In addition, pregnancy is considered a leading cause of the lack of physical activities in women $(5,6)$. Many women take a sedentary lifestyle during pregnancy and compared to before pregnancy, they reduce the frequency, duration and intensity of their physical activities due to the fear of injuries to the fetus (7). According to studies conducted in the USA, $60 \%$ of pregnant women take a sedentary lifestyle (8). The American Congress of Obstetricians and Gynecologists and the College of Sports Medicine, recommend that pregnant women who have no particular obstetric complications can have at least 30 minutes of moderate physical activities in the day provided that traumatic activities are avoided $(9,10)$. Sufficient and correct Physical activities have positive effects on mothers' health and fetal growth (11) including the reduction of the prevalence of hypertension, preeclampsia and eclampsia $(1,12)$. Developing the feeling of wellbeing, increasing self-esteem, improving body image, decreasing anxiety and depression, quick and easy adaptation to changes caused by pregnancy influence doing exercise during pregnancy (13). While physical activities help achieve the desired pregnancy outcomes, measuring physical activities is a difficult and complex process (14). A reliable and valid method is required for measuring the duration, frequency and intensity of physical activities. It helps to design appropriate recommendations, cross-cultural comparisons and assess the effects of physical activities with various intensities on the health of pregnant women. The easiest and most common methods for measuring physical activities are questionnaire-based self-report and interview $(15,16)$. Some questionnaires about physical activities have been validated among non-pregnant adults. They mostly have not considered physical activities such as housework and childcare issues and were not tailored to pregnancy $(16,17)$. An inaccurate assessment of the levels of physical activities during pregnancy leads to an inappropriate understanding of the relationship between 
physical activities and health during pregnancy (15). The pregnancy physical activity questionnaire is an instrument for assessing and measuring the amount of physical activities in pregnancy and studying the intensity, duration and frequency of physical activity among pregnant women $(1,18)$. This questionnaire has been translated into other languages (18). It was first compiled in the United States by Chasan-Taber et al $(15,19)$. The reliability of this instrument has been assessed using Cronbach's alpha coefficient and reported to be 0.83 for the whole instrument and $0.72-0.85$ for domains $(1,15$, 16). It was stated that race, ethnicity, lifestyle habits and cultural beliefs of the society affected the amount of physical activities during pregnancy (20). Given the importance of physical activities among pregnant women and the need for urgent interventions in case of problems during pregnancy and lack of a standard scale, the purpose of this study was to evaluate the psychometric properties of the Persian version of the pregnancy physical activity questionnaire.

\section{Materials and Methods}

Study Design and Participants

This cross-sectional methodological study was conducted on pregnant women referred to healthcare centers in Tabriz from October to January 2016. The place of the study as the fifth largest city in Iran had a population of more than 1.5 million people. It had 42 healthcare centers and 33 healthcare stations. Inclusion criteria were as follows: being Iranian, low-risk and singleton pregnancy, the age range of 20-40 years and having the ability to read and write in Persian. Exclusion criteria were the diagnosis of chronic disorders during pregnancy such as diabetes, cardiovascular diseases, lung and kidney diseases and high blood pressure, the risk of preterm childbirth, which could limit the movement of pregnant women. The required sample size for conducting factor analysis was considered 5 women per the questionnaire items $(21,22)$ but some assume that a sample size of 200 is adequate (23). Based on the number of items in the questionnaire, 150 samples were calculated but sampling continued until achieving a sample size of 200 to complete the full questionnaire. Using a multi-stage method, healthcare centers and healthcare stations were chosen via a software (https:// www.random.org) (24). Given the number of pregnant women in healthcare centers and healthcare stations, the number of samples in each center was calculated based on their proportion in each center or station. The researcher (AFK) referred to the healthcare centers, selected qualified women and extracted information pertaining to women's weight and height. Next, questionnaires were collected using the self-report method. The average time to fill out the questionnaire by the women was 10 to 15 minutes.

Study Instrument

Designed by Chasan-Taber et al, the pregnancy physical activity questionnaire consists of 2 parts (15). The first part has three questions about the date of completing the form, date of the first day of the last menstruation and approximate date of pregnancy. The second part consists of 33 items in four areas of transportation (3 items), activity at home and caring issues (16 items), recreational and sports activities ( 9 items) and career activity ( 5 items). The time spent on any kind of physical activity was calculated on the basis of the metabolic equivalent test (MET). Sedentary activity, light activity, moderate activity and intense activity are regarded as less than 1.5 Met, 1.5-2.9 Met, 3-6 Met and more than 6 Met, respectively $(15,25)$. Information concerning the most important demographic and obstetric characteristics of the patients was asked at the beginning of the questionnaire.

\section{Data Analysis \\ Translation Into Persian}

After obtaining permission from the questionnaire designer, the translation process was conducted according to the approach used by Jones et al (26). The questionnaire was translated from English into Persian independently by 2 experts in the field of reproductive health. A Farsi version was created by comparing the 2 translated versions. It helped clarify and reach a common understanding by the readers of the questionnaire. A reverse translation from Farsi into English was conducted by 2 translators who were unaware of the content of the original questionnaire. Next, the new English version was compared with the original questionnaire. After approval of the translation, a final version of the questionnaire was provided.

\section{Face Validity}

Face validity is a method for subjective judgments about the validity of an instrument $(27,28)$. The face validity of this questionnaire was assessed using 2 qualitative and quantitative methods. The questionnaire was given to 20 pregnant women referred to healthcare centers and they were asked to assess it in terms of difficulty levels (phrases or words), level of appropriateness (suitability and proper relationships between the phrases of the questionnaire) and ambiguity (the possibility of misinterpretations of phrases and meanings). The quantitative stage aimed to eliminate or reduce similar phrases and determine the degree of importance of each phrase. The impact factor for each item of the questionnaire was based on the fivepoint Likert scale indicating the degree of the importance of each item. If the impact score was more than 1.5, the items were maintained and found suitable for further analysis (26).

\section{Content Validity}

For content validity, 10 experts in the field of reproductive health, obstetrics and gynecology participated. The content validity ratio (CVR) and content validity index (CVI) were calculated for the quantitative assessment of 
content validity. For calculating the CVR, the women were asked to assess the necessity of each item using a 5-point Likert scale (it is necessary, it is useful but not necessary and it is not necessary). According to Lawshe's table (29), if the score of the item was more than or equal to 0.62 , the item was maintained in the questionnaire (30).

\section{Construct Validity}

It refers to the adequacy of an instrument for determining the instrument structure and organizing items (31). Exploratory factor analysis and varimax rotation were used to assess construct validity $(21,22)$. Applying exploratory factor analysis is helpful when researchers cannot find sufficient reasons for categorizing items and the relationship between factors. If the factor loading was equal or more than 0.4 , it was considered significant. The KMO index (Kaiser-Mayer-Olkin) and Bartlett's test of sphericity helped assess the adequacy of sampling and determine correlation matrix. The significance level was set at $P<0.05(22)$.

\section{Reliability}

The test-retest method, Cronbach's alpha coefficient and intra-class correlation coefficient (ICC) were used to assess reliability $(32,33)$. Within a 2 -week interval, the researchers gave the tool to 20 qualified women participating in the study. The scores in the second stage were compared using the ICC. In addition, the internal consistency of the tool was evaluated for each factor using Cronbach's alpha coefficient. An alpha coefficient equal or more than 0.7 was considered satisfactory. Furthermore, the ICC less than 0.4 was weak, 0.41-0.6 was medium, 0.61- 0.8 was good and above 0.8 was considered excellent.

\section{Data Analysis}

Descriptive (mean and standard deviations, frequency and percentage) and interpretive statistics (factor analysis) were used for data analysis via the SPSS software version 19.0. A $P$-value less than 0.05 was considered statistically significant.

\section{Results}

After data collection, 3 questionnaires, which were partially completed, were replaced with three other samples. It was found that 134 women $(67 \%)$ were 20 -30 years old with the mean gestational age of $28.20 \pm 8.53$ weeks and mean body mass index (BMI) at the beginning and during pregnancy was $25.46 \pm 3.73,28.87 \pm 3.86$, respectively. Moreover, the mean weight gain during pregnancy was $8.63 \pm 4.85 \mathrm{~kg}$. Table 1 shows the demographic and obstetric characteristics of the samples.

Furthermore, the average physical activities in terms of MET was reported to be 41.31 (Table 2).

During the evaluation of the content validity, the viewpoints of experts led to slight changes in some items. The CVR and CVI were reported to be $0.2-1.00$ and 0.8-
Table 1. The Demographic and Obstetrics Characteristics of the Samples

\begin{tabular}{|c|c|}
\hline Variable & Mean \pm SD \\
\hline Pregnant woman's age & $28.55 \pm 5.04$ \\
\hline \multirow[t]{2}{*}{ Gestational age based on LMP (wk) } & $28.20 \pm 8.53$ \\
\hline & No. (\%) \\
\hline \multicolumn{2}{|l|}{ Number of gestations } \\
\hline 1 & $69(34.5)$ \\
\hline 2 & $81(40.5)$ \\
\hline$\geq 3$ & $40(25)$ \\
\hline \multicolumn{2}{|l|}{ Number of childbirths } \\
\hline Zero & $82(41)$ \\
\hline 1 & $92(46)$ \\
\hline$\geq 2$ & $26(13)$ \\
\hline \multicolumn{2}{|l|}{ Trimester } \\
\hline The first & $24(12)$ \\
\hline The second & $65(32.5)$ \\
\hline The third & $111(55.5)$ \\
\hline \multicolumn{2}{|l|}{$\mathrm{BMI}$ at the beginning of pregnancy } \\
\hline$<18.5$ & $6(3)$ \\
\hline $18.5-24.9$ & $92(46)$ \\
\hline $25-29.9$ & $77(38.5)$ \\
\hline$>30$ & $25(12.5)$ \\
\hline \multicolumn{2}{|l|}{ BMI during pregnancy } \\
\hline $18.5-24.9$ & $30(15)$ \\
\hline $25-29.9$ & $91(45.5)$ \\
\hline$>30$ & $79(39.5)$ \\
\hline \multicolumn{2}{|l|}{ Occupation } \\
\hline Housewife & $169(85.5)$ \\
\hline Employed & $31(15.5)$ \\
\hline \multicolumn{2}{|l|}{ Education level } \\
\hline Primary school & $20(10)$ \\
\hline Secondary school & $24(12)$ \\
\hline High school & $97(48.5)$ \\
\hline College/university & $59(29.5)$ \\
\hline
\end{tabular}

Table 2. The Man and Standard Deviation of Physical Activities on MET

\begin{tabular}{lc}
\hline Type of activity & Mean \pm SD \\
\hline Total activity & $41.31 \pm 19.8$ \\
Household/caregiving & $33.19 \pm 17.33$ \\
Transportation & $3.19 \pm 2.74$ \\
Sports/exercise & $3.30 \pm 3.83$ \\
Occupational activity & $1.73 \pm 5.48$ \\
\hline
\end{tabular}

1.00 , respectively. In addition, phrases 15 and 16 were combined and changed to 'activities related to gardening and planting'

Following face and content validity, 30 items were entered into exploratory factor analysis. The KMO index ratio for the assessment of the adequacy of data and the correlation matrix was reported to be 0.654 . Therefore, the sample size was sufficient to calculate factor analysis. Furthermore, the Bartlett test with the ratio of 1.826 indicated that 
factor analysis for identifying the structure of the factor model was significant $(P>0.001)$. Relationships between the variables included in factor analysis were found. The second output of exploratory factor analysis consisted of 2 initial special values and special quantities of extracted factors with rotation (Table 3 ).

After obtaining extrapolating values higher than one and using the scree plot (Figure 1), a 5-factor model was developed: 'vulnerable people, 'activities related to work', 'home', 'sports' and 'activities related to leisure time. They accounted for $46.324 \%$ of the observed variance.

The first factor consisted of 6 items with the factor loading ranging from 0.588 to 0.805 . The second factor consisted of 5 items with a minimum factor loading of 0.488-0.910. The third factor consisted of 7 items that showed a factor loading ranging from 0.318 to 0.577 . The fourth factor had 10 items with the minimum factor loading of 0.31 and the maximum of 0.655 . The fifth factor had 2 items with the factor loading of 0.481-0.659. Factor loadings related to the 30-question final model were shown in Table 3. According to the results of this table, the estimation of all standardized factor loadings was reported to be 0.05 (Table 3).

Reliability

The Cronbach's alpha ratios were presented for the total

Table 3. The Rotated Matrix of Factor Analysis Components of the Pregnancy Physical Activity Questionnaire

\begin{tabular}{|c|c|c|c|c|c|c|c|c|}
\hline \multirow{2}{*}{ Factors } & \multirow{2}{*}{$\begin{array}{l}\text { Items of Menopausal Symptoms Assessment } \\
\text { Instrument }\end{array}$} & \multirow{2}{*}{$\begin{array}{l}\text { Factor } \\
\text { Loading }\end{array}$} & \multicolumn{3}{|c|}{ Initial Eigenvalues } & \multicolumn{3}{|c|}{ Rotation Sums of Loading } \\
\hline & & & Total & $\%$ & Cumulative\% & Total & $\%$ & Cumulative\% \\
\hline \multirow{6}{*}{$\begin{array}{l}\text { Factor } 1 \text { Caring for } \\
\text { vulnerable people }\end{array}$} & $\begin{array}{l}\text { Dressing, bathing, feeding children while you are } \\
\text { sitting; }\end{array}$ & 0.805 & 4.267 & 14.715 & 14.715 & 3.614 & 12.461 & 12.461 \\
\hline & $\begin{array}{l}\text { Dressing, bathing, feeding children while you are } \\
\text { standing; }\end{array}$ & 0.793 & & & & & & \\
\hline & $\begin{array}{l}\text { Playing with children while you are sitting or } \\
\text { standing; }\end{array}$ & 0.8 & & & & & & \\
\hline & $\begin{array}{l}\text { Playing with children while you are Walking or } \\
\text { running; }\end{array}$ & 0.709 & & & & & & \\
\hline & Carrying children; & 0.763 & & & & & & \\
\hline & Taking care of an older adult & 0.588 & & & & & & \\
\hline \multirow{5}{*}{$\begin{array}{l}\text { Factor } 2 \text { Job-related } \\
\text { activities }\end{array}$} & Sitting at work or in class; & 0.847 & 2.935 & 10.121 & 24.837 & 2.927 & 10.092 & 22.553 \\
\hline & $\begin{array}{l}\text { Standing or slowly walking at work, while carrying } \\
\text { things (heavier than a 1-gallon milk jug); }\end{array}$ & 0.692 & & & & & & \\
\hline & $\begin{array}{l}\text { Standing or slowly walking at work, not carrying } \\
\text { anything; }\end{array}$ & 0.910 & & & & & & \\
\hline & $\begin{array}{l}\text { Walking quickly at work while carrying things } \\
\text { (heavier than a 1-gallon milk jug); }\end{array}$ & 0.640 & & & & & & \\
\hline & Walking quickly at work not carrying anything & 0.488 & & & & & & \\
\hline \multirow{7}{*}{$\begin{array}{l}\text { Factor } 3 \text { Household } \\
\text { activity }\end{array}$} & Preparing meals (cook, set table, wash dishes); & 0.54 & 2.678 & 9.236 & 34.072 & 2.679 & 9.239 & 31.793 \\
\hline & $\begin{array}{l}\text { Sitting and using a computer or writing, while not } \\
\text { at work; }\end{array}$ & 0.318 & & & & & & \\
\hline & Watching TV or a video; & 0.438 & & & & & & \\
\hline & $\begin{array}{l}\text { Sitting and reading, taking or on the phone, while } \\
\text { not at work; }\end{array}$ & 0.360 & & & & & & \\
\hline & $\begin{array}{l}\text { Light cleaning (make beds, Laundry, Iron, put } \\
\text { things away); }\end{array}$ & 0.539 & & & & & & \\
\hline & Shopping (for food, clothes, or other items); & 0.426 & & & & & & \\
\hline & $\begin{array}{l}\text { Having cleaning (vacuum, mop, sweep, wash } \\
\text { windows) }\end{array}$ & 0.577 & & & & & & \\
\hline \multirow{10}{*}{$\begin{array}{l}\text { Factor } 4 \text {. Sports } \\
\text { activities and } \\
\text { commuting }\end{array}$} & $\begin{array}{l}\text { Walking slowly to go places (such as to the bus, } \\
\text { work, visiting); }\end{array}$ & 0.655 & 1.985 & 6.844 & 40.917 & 1.487 & 8.575 & 40.368 \\
\hline & $\begin{array}{l}\text { Walking quickly to go places (such as to the bus, } \\
\text { work, school); }\end{array}$ & 0.31 & & & & & & \\
\hline & Driving; & 0.34 & & & & & & \\
\hline & Walking slowly for fun or exercise; & 0.32 & & & & & & \\
\hline & Walking more quickly for fun or exercise; & 0.649 & & & & & & \\
\hline & Walking quickly up hills for fun or exercise; & 0.648 & & & & & & \\
\hline & Jogging; & 0.515 & & & & & & \\
\hline & Prenatal exercise class; & 0.618 & & & & & & \\
\hline & Swimming; & 0.652 & & & & & & \\
\hline & Dancing & 0.432 & & & & & & \\
\hline $\begin{array}{l}\text { Factor } 5 \text { Activities } \\
\text { related to leisure } \\
\text { time }\end{array}$ & $\begin{array}{l}\text { Playing with animal; } \\
\text { Activities related to planting and gardening }\end{array}$ & $\begin{array}{l}0.659 \\
0.481\end{array}$ & 1.568 & 5.407 & 46.324 & 1.727 & 5.956 & 46.324 \\
\hline
\end{tabular}




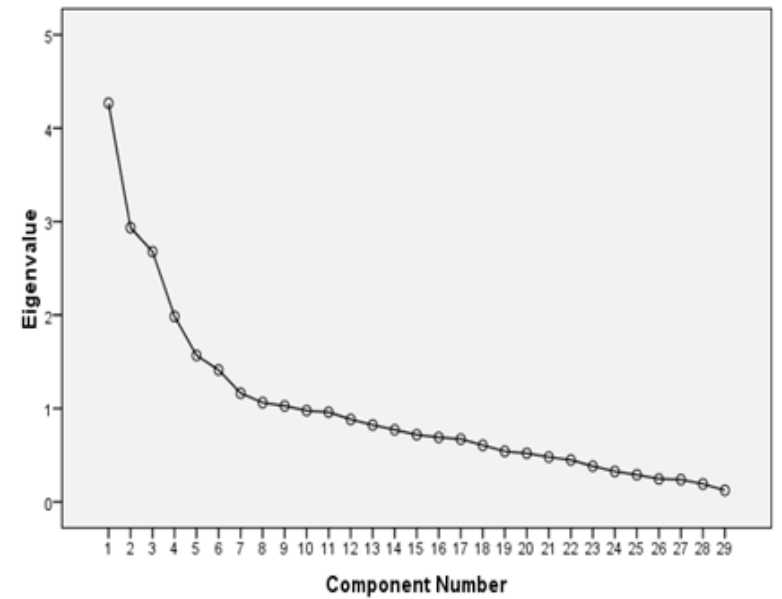

Figure 1. The Scree Plot Based on Factor Analysis to Assess the Correlation Between Items.

and individual factors in Table 4. The Cronbach alpha coefficient of the entire questionnaire was reported to be 0.879 , indicating that only $10 \%$ of the variance of the questionnaire scores was achieved by errors in measurements. The ICC for the entire questionnaire was 1.857 (95\% CI $=0.75-0.93$ ). Additionally, for studying the convergence of the items, the Cronbach's alpha coefficients for all items were calculated. The alpha of 0.9 showed that none of the questions can be removed. Therefore, all items remained in the instrument.

\section{Discussion}

Epidemiological studies need an instrument that takes less time and energy to be completed (34). Iranian women often are involved in housekeeping activities. Therefore, this instrument is useful for data collection regarding the amount of physical activities in Iranian women. In the present study, the psychometric properties of the pregnancy physical activity questionnaire were assessed after its translation into Farsi. During the evaluation of content validity, items 15 and 16 were merged for adaptation to Iranian culture and context. The number of items was reduced to 30 items, which is similar to the number of items in the study by Tosun et al in Turkey (10). In a study carried out in Japan, one item called 'cycling' was added to the questionnaire in the domain of sports activity, which was for the transportation of pregnant women (25). Factor analysis indicated a 5-factor solution due to the cultural aspects of Iranian society. Unlike the original scale, factor loading of some items was different from the original version. For instance, items 6, 5, 4, 3, 2, and 7 were loaded on the caring factor in the vulnerable people, and items 15 and 18 were loaded on activities related to leisure time. Moreover, sports activities and transportation were located under one factor. However, the results of factor analysis showed that total factor structure was consistent with that in the original version. In addition, it was indicated that the pregnancy physical activity questionnaire was reliable for the measurement of physical activities. The Cronbach $\alpha$ coefficients for the domains were at an acceptable level. In a study in Turkey, Tosun et al found similar results and reported Cronbach's alpha coefficients to be 0.70-0.95 (10). In addition, the internal clustering coefficient over 0.8 for the entire questionnaire indicated high reliability in this study. In the study of Chasan-Taber et al., the correlation coefficient for the entire activity was 0.78 (15). The validity and reliability of the pregnancy physical activity questionnaire among obese pregnant women were evaluated by Chandonnet et al in France. They reported the correlation coefficient of 0.9 for the entire activity. They concluded that the pregnancy physical activity questionnaire was reliable to measure physical activities with different intensities among obese pregnant women (35). Furthermore, the results of the study by Ota et al in Vietnam on 60 healthy pregnant women with singleton pregnancies showed that the correlation coefficient was 0.88 for the entire activity (16). Therefore, Turkish, French, and Vietnamese versions of this questionnaire had great internal clustering coefficients, which were similar to this study (1).

Moreover, after calculating the ratio of the entire activity and domains of the questionnaire as hours spent for each activity with the Met value for the same activities (36) and sum of all activities, the entire levels of physical activities of the women were found to be lower than the results of the study by Chirak et al in Turkey (1). However, our result was consistent with those of the study conducted by Ota et al (16). Among the scores of physical activities, the highest score was for household activities with a mean of 33.19. It was reported to be 38 Met in the study of Utah et al (16). Nevertheless, it was reported to be 10.6 in the United States even though American women achieved higher scores for physical activities (19). The women in this study had low scores concerning other activities especially in sports and

Table 4. Correlation, Reliability and Internal Consistency of the Domains of Questionnaire

\begin{tabular}{|c|c|c|c|c|c|c|}
\hline Factor & Mean & SD & Cronbach $\alpha$ & ICC Coefficient & $95 \% \mathrm{Cl}$ & No. of Questions \\
\hline Factor 1: Caring for vulnerable people & 6.45 & 5.726 & 0.715 & 0.725 & $0.510-0.873$ & 7 \\
\hline Factor 2: Job-related activities & 2.95 & 5.568 & 0.936 & 0.882 & $0.882-0.971$ & 5 \\
\hline Factor 3: Household activity & 14.50 & 4.059 & 0.886 & 0.884 & $0.795-0.945$ & 6 \\
\hline Factor 4: Sports activities and commuting & 4.95 & 3.993 & 0.877 & 0.865 & $0.760-0.937$ & 10 \\
\hline Factor 5: Activities related to leisure time & 0.85 & 1.565 & 0.94 & 0.936 & $0.873-0.972$ & 2 \\
\hline
\end{tabular}

Abbreviation: ICC, intra-class correlation. 
recreational fields.

\section{Conclusions}

The Persian version of the pregnancy physical activity questionnaire is a valid and reliable tool for examining the risk of physical activities among Iranian pregnant women so it can be used in epidemiological studies.

\section{Conflict of Interests}

Authors declare that they have no conflict of interests.

\section{Ethical Issues}

The research council affiliated with the University of Medical Sciences in which the authors worked approved the study proposal (registration code: IR. SBMU.PHNM.1395.666). Verbal consent was obtained from the pregnant woman after assuring them of the preservation of their confidentiality and possibility of withdrawal from the study without being penalized. Next, the women were asked to fill out the pregnancy physical activity questionnaire.

\section{Financial Support}

Deputy of Research, Shahid Beheshti University of Medical Sciences, Tehran, Iran supported our study.

\section{Acknowledgments}

The authors of this article would like to appreciate Dr. Chasan for giving permission to translate and use the questionnaire. We also would like to thank the research deputy of Shahid Beheshti University of Medical Sciences, Tabriz University of Medical Sciences, authorities and staff of healthcare centers and stations and the women who helped us with the publication of this article.

\section{References}

1. Cirak Y, Yilmaz GD, Demir YP, Dalkilinc M, Yaman S. Pregnancy physical activity questionnaire (PPAQ): reliability and validity of Turkish version. J Phys Ther Sci. 2015;27(12):3703-3709. doi:10.1589/jpts.27.3703

2. Garber CE, Blissmer B, Deschenes MR, et al. American College of Sports Medicine position stand. Quantity and quality of exercise for developing and maintaining cardiorespiratory, musculoskeletal, and neuromotor fitness in apparently healthy adults: guidance for prescribing exercise. Med Sci Sports Exerc. 2011;43(7):1334-1359. doi:10.1249/MSS.0b013e318213fefb

3. Physical Activity Guidelines Advisory Committee (PAGAC). Physical Activity Guidelines Advisory Committee Report, 2008. Washington, DC: PAGAC; 2008.

4. Department of Health and Human Services, 2008. O'Donovan G, Blazevich AJ, Boreham C, et al. The ABC of Physical Activity for Health: a consensus statement from the British Association of Sport and Exercise Sciences. J Sports Sci. 2010;28(6):573-591. doi:10.1080/02640411003671212

5. Evenson KR. Towards an Understanding of Change in Physical Activity from Pregnancy Through Postpartum. Psychol Sport Exerc. 2011;12(1):36-45. doi:10.1016/j. psychsport.2010.04.010

6. Akbari Z, Tol A, Shojaeizadeh D, Aazam K, kia F. Assessing of physical activity self-efficacy and knowledge about benefits and safety during pregnancy among women. Razi
Journal of Medical Sciences. 2016;22(139):76-87.

7. Reid H, Smith R, Calderwood C, Foster C. Physical activity and pregnancy: time for guidance in the UK. Br J Sports Med. 2017;51(21):1511-1512. doi:10.1136/bjsports-2016-097216

8. Symons Downs D, Hausenblas HA. Women's exercise beliefs and behaviors during their pregnancy and postpartum. J Midwifery Womens Health. 2004;49(2):138144. doi:10.1016/j.jmwh.2003.11.009

9. Shakeri M, Fekri S, Shahnavaz A, Shakibazadeh E. Effectiveness of a group-based educational program on physical activity among pregnant women. Journal of Hayat. 2012;18(3):1-9.

10. Tosun OC, Solmaz U, Ekin A, et al. The Turkish version of the pregnancy physical activity questionnaire: crosscultural adaptation, reliability, and validity. J Phys Ther Sci. 2015;27(10):3215-3221. doi:10.1589/jpts.27.3215

11. Cohen TR, Plourde H, Koski KG. Use of the Pregnancy Physical Activity Questionnaire (PPAQ) to identify behaviours associated with appropriate gestational weight gain during pregnancy. J Phys Act Health. 2013;10(7):10001007. doi:10.1123/jpah.10.7.1000

12. Kramer MS, McDonald SW. Aerobic exercise for women during pregnancy. Cochrane Database Syst Rev. 2006(3):Cd000180. doi:10.1002/14651858.CD000180.pub2

13. Exercise during pregnancy and the postpartum period. Clin Obstet Gynecol. 2003;46(2):496-499.

14. Helmerhorst HJ, Brage S, Warren J, Besson H, Ekelund U. A systematic review of reliability and objective criterionrelated validity of physical activity questionnaires. Int J Behav Nutr Phys Act. 2012;9:103. doi:10.1186/1479-58689-103

15. Chasan-Taber L, Schmidt MD, Roberts DE, Hosmer D, Markenson G, Freedson PS. Development and validation of a Pregnancy Physical Activity Questionnaire. Med Sci Sports Exerc. 2004;36(10):1750-1760. doi:10.1249/01. MSS.0000142303.49306.0D

16. Ota E, Haruna M, Yanai H, et al. Reliability and validity of the Vietnamese version of the Pregnancy Physical Activity Questionnaire (PPAQ). Southeast Asian J Trop Med Public Health. 2008;39(3):562-570.

17. Schneider PL, Crouter S, Bassett DR. Pedometer measures of free-living physical activity: comparison of 13 models. Med Sci Sports Exerc. 2004;36(2):331-335. doi:10.1249/01. mss.0000113486.60548.e9

18. Mehran N, Abaszadeh A, Bageri A. The relationship between home work and preterm labor. Hayat. 2013;18(5):46-54. [Persian].

19. Chasan-Taber L, Evenson KR, Sternfeld B, Kengeri S. Assessment of recreational physical activity during pregnancy in epidemiologic studies of birthweight and length of gestation: methodologic aspects. Women Health. 2007;45(4):85-107. doi:10.1300/J013v45n04_05

20. Jonsson LS, Palmer K, Ohlsson H, Sundquist J, Sundquist $\mathrm{K}$. Is acculturation associated with physical activity among female immigrants in Sweden? J Public Health (Oxf). 2013;35(2):270-277. doi:10.1093/pubmed/fds091

21. Bryant FB, Yarnold PR. Principal-components analysis and exploratory and confirmatory factor analysis. In: Grimm LG, Yarnold PR, eds. Reading and understanding multivariate statistics. Washington, DC, US: American 
Psychological Association; 1995:99-136.

22. Tinsley HE, Brown SD. Handbook of applied multivariate statistics and mathematical modeling. Academic Press; 2000.

23. Salganik MJ. Variance estimation, design effects, and sample size calculations for respondent-driven sampling. J Urban Health. 2006;83(6 Suppl):i98-112. doi:10.1007/ s11524-006-9106-x

24. Haahr M. Random. org: true random number service list randomizer. 2011. Available from: https://www.random. org/.

25. Matsuzaki M, Haruna M, Nakayama K, et al. Adapting the Pregnancy Physical Activity Questionnaire for Japanese pregnant women. J Obstet Gynecol Neonatal Nurs. 2014;43(1):107-116. doi:10.1111/1552-6909.12267

26. Jones EG, Mallinson RK, Phillips L, Kang Y. Challenges in language, culture, and modality: translating English measures into American sign language. Nurs Res. 2006;55(2):75-81.

27. Drost EA. Validity and reliability in social science research. Education Research and Perspectives. 2011;38(1):105-124.

28. Roberts P, Priest H, Traynor M. Reliability and validity in research. Nurs Stand. 2006;20(44):41-45. doi:10.7748/ ns2006.07.20.44.41.c6560

29. Lawshe $\mathrm{CH}$. A quantitative approach to content validity 1. Pers Psychol. 1975;28(4):563-575. doi:10.1111/j.1744-6570.1975.tb01393.x

30. Waltz CF, Bausell RB. Nursing research: design, statistics, and computer analysis. Philadelphia: F.A. Davis Co; 1981.

31. Polit DF, Beck CT. Essentials of nursing research: Appraising evidence for nursing practice. Baltimore: Lippincott Williams \& Wilkins; 2013.

32. Bartko JJ. The intraclass correlation coefficient as a measure of reliability. Psychol Rep. 1966;19(1):3-11. doi:10.2466/ pr0.1966.19.1.3

33. Cronbach LJ. Coefficient alpha and the internal structure of tests. Psychometrika. 1951;16(3):297-334. doi:10.1007/ bf02310555

34. Yousefi R. Psychometric properties of Persian version of renataldistressquestionnaire (PDQ). Journal of Urmia Nursing and Midwifery Faculty. 2015;13(3):215-225. [Persian].

35. Chandonnet N, Saey D, Almeras N, Marc I. French Pregnancy Physical Activity Questionnaire compared with an accelerometer cut point to classify physical activity among pregnant obese women. PLoS One. 2012;7(6):e38818. doi:10.1371/journal.pone.0038818

36. Ainsworth BE, Haskell WL, Whitt MC, et al. Compendium of physical activities: an update of activity codes and MET intensities. Med Sci Sports Exerc. 2000;32(9 Suppl):S498-504.

(c) 2019 The Author (s); This is an open-access article distributed under the terms of the Creative Commons Attribution License (http://creativecommons.org/licenses/by/4.0), which permits unrestricted use, distribution, and reproduction in any medium, provided the original work is properly cited. 